\title{
MODELOS ESTATÍSTICOS E DE REDES NEURAIS ARTIFICIAIS UTILIZADOS NA PREDIÇÃO DE PRECIPITAÇÃO
}

\author{
Ronaldo Toshiaki Oikawa ${ }^{1}$
}

\section{Hamilton Mitsugu Ishiki ${ }^{2}$}

RESUMO: A interferência do homem pode acelerar, em muito, os processos naturais e as mudanças climáticas trazendo graves consequências à vida na terra. De forma específica, alguns fatores e elementos do meio natural são determinantes para a existência e sobrevivência de indivíduos ou de grupos taxonômicos, como por exemplo, a chuva. $\mathrm{O}$ aproveitamento adequado dos recursos hídricos requer o conhecimento das precipitações prováveis capazes de ocorrer em uma determinada localidade. Isto é possível por meio do uso de modelos matemáticos de probabilidade ou de redes neurais artificiais. Neste contexto, o presente trabalho apresenta os principais modelos probabilísticos e de redes neurais artificiais utilizados atualmente para a predição da precipitação, como sendo parte importante do ciclo hidrológico no planeta Terra.

Palavra chave: Precipitação pluvial, Redes neurais artificias, Modelos estatísticos.

\section{INTRODUÇÃO}

As mudanças climáticas que estão ocorrendo em nosso planeta, em especial os fenômenos relacionados com as chuvas, têm atraído consideráveis esforços na quantidade de pesquisa, principalmente por causa da ocorrência de enchentes e os danos a ela relacionados (KATTSOV et al., 2007; HOLMAN e VAVRUS, 2012).

\footnotetext{
${ }^{1}$ Discente do Programa de Mestrado em Meio Ambiente e Desenvolvimento Regional da Universidade do Oeste Paulista - ot.ronaldo@gmail.com

${ }^{2}$ Docente do Programa de Mestrado em Meio Ambiente e Desenvolvimento Regional da Universidade do Oeste Paulista - hamilton@unoeste.br
} 


\section{S Fen Fórum Ambiental \\ da Alta Paulista

As simulações adequadas de modelos climáticos são muito importantes para 0 ser humano, como por exemplo, para se evitar tragédias, realizar o planejamento de recursos hídricos, para estudos hidrológicos, tomar decisões em relação à geração de energia elétrica e algumas atividades agrícolas e industriais (QUEIROZ, SILVA e OLIVEIRA, 2001; SAMPAIO et al., 2007; ÁVILA, MELLO e VIOLA, 2009).

A precipitação pluviométrica pode ser prevista através de modelos teóricos probabilísticos de distribuição, ajustados a uma série de dados. Para serem confiáveis, estes modelos de simulação climáticos devem possuir uma grande variedade de parâmteros observados. Devido à grande quantidade de parâmetros necessários, alguns modelos podem assumir diferentes formas, podendo ser utilizados em um número maior de situações (NETO et al., 2005). Ao se utilizar projeções baseadas em modelos validados, decisões e análises, as predições climáticas podem ser realizadas com maior confiança (KAWAZOE e GUTOWSKI JR., 2013). Entretanto, um dos maiores inconvenientes nos modelos de simulação que envolve a ocorrência de precipitações é a sua incapacidade de reconhecê-las em sua forma real. Esta restrição ocorre quando o modelo ignora ou não distingue a variabilidade da distribuição temporal da chuva, ou seja, o seu tempo de duração (CRUCIANI, MACHADO e SENTELHAS, 2002).

$\mathrm{Na}$ literatura é possível encontrar vários modelos probabilísticos teóricos para a realização do cálculo de precipitação esperada (CRUCIANI, MACHADO e SENTELHAS, 2002; MOREIRA et al., 2010). Os atuais modelos de previsão numérica de tempo conseguem prever com certo grau de exatidão a precipitação. Entretanto, a física associada à sua análise é muito complexa (OLSON, JUNKER e KORTY, 1995). A complexidade física dos processos de precipitação não pode ser resolvida, até o presente momento, pelos modelos numéricos existentes. Mesmo os modelos mais sofisticados de previsão numérica de tempo não conseguem por meio de suas parametrizações estimar satisfatoriamente o campo de chuva (OLSON, JUNKER e KORTY, 1995).

$\mathrm{Na}$ tentativa de se superar as deficiências dos modelos matemáticos tradicionais, existem na literatura trabalhos que utilizam modelos baseados em redes neurais artificiais (BILGILI e SAHIN, 2010; WU, CHAU e FAN, 2010; WU e CHAU, 2013). Esta técnica permite estabelecer relações não lineares entre a precipitação observada, as saídas de um modelo de previsão numérica de tempo e dados obtidos por satélite (HORNIK, STINCHCOMBE e WHITE, 1989). 
O presente trabalho irá mostrar as metodologias utilizadas para a realização da predição de chuva tanto através dos principais modelos matemáticos probabilísticos, bem como através das redes neurais artificiais.

\section{MODELOS PROBABILÍSTICOS}

\subsection{Distribuição Gama}

THOM (1958) e CUNHA, MARTINS e PASSOS (1996) consideram que, dentre os modelos probabilísticos avaliados por meio de análises em histogramas de frequência, $\mathrm{O}$ modelo da distribuição Gama é o que tem apresentado melhores resultados, em estimativa de probabilidades e na simulação de dados climáticos. Uma das vantagens em utilizar a distribuição gama é a possibilidade de trabalhar com períodos mensais ou menores como, por exemplo, decêndios e quinzenas (ASSIS, ARRUDA e PEREIRA, 1996).

A distribuição Gama com dois parâmetros é um caso especial de distribuição de Pearson Tipo II no qual o parâmetro local é definido como sendo zero. Na literatura podem ser encontradas uma vasta série de estudos que indicam a distribuição gama, como sendo o meio probabilístico mais confiável (MURTA et al., 2005, SILVA et al., 2007, SAMPAIO et al., 2007; LIMA et al., 2008).

A equação densidade de probabilidade para a distribuição Gama é dada pela equação 1.

$$
f(x)=\frac{1}{\beta^{\alpha} \Gamma(\alpha)} x^{\alpha-1} e^{\frac{-x}{\beta}}
$$

onde: $\beta$ = parâmetro de escala $(\mathrm{mm}) ; \alpha=$ parâmetro de forma (adimensional); $\mathrm{e}=$ base do logaritmo neperiano; $x=$ total de precipitação $(\mathrm{mm})$ e $\Gamma(\alpha)=$ função Gama, onde esta é dada pela equação 2 .

$$
\Gamma(\alpha)=\sqrt{2 \pi \alpha} \alpha^{\alpha} e^{\alpha}\left(1+\frac{1}{12 \alpha}+\frac{1}{288 \alpha^{2}}-\frac{139}{51840 \alpha^{3}}\right)
$$


Sendo que os parâmetros que propiciam a distribuição Gama acumulada, para certa variável aleatória ( $\alpha$ e $\beta$ ), foram estimados pelo método dos momentos, descrito por Thom (1958):

$$
\alpha=\frac{\bar{X}^{2}}{S^{2}} \text { e } \beta=\frac{S^{2}}{\bar{X}}
$$

Um grande problema na utilização da distribuição Gama é a estimativa de seus parâmetros $\alpha$ e $\beta$ cujos cálculos são complexos e extensos.

\subsection{Distribuição log-normal}

$\mathrm{Na}$ distribuição Log-normal é considerada que a distribuição de variáveis aleatórias tem a propriedade em que os logaritmos dos seus valores sejam normalmente distribuídos, sendo a função densidade normal dada por:

$$
f(x ; \mu, \sigma)=\frac{1}{x \sigma \sqrt{2 \pi}} \exp \left[-\frac{(\ln (x)-\mu)^{2}}{2 \sigma^{2}}\right]
$$

equação 3

A distribuição Log-normal possuí uma distribuição unilateral, ou seja, possui valores apenas no intervalo positivo. Esta propriedade é conveniente para se trabalhar com propriedades físicas que só possuem valores positivos. Além de ser fácil de ser utilizada, esta distribuição tem-se ajustado bem à distribuição pluviométrica brasileira (SAMPAIO et al., 1999; RODRIGUES e PRUSKI, 2001).

\subsection{Distribuição de precipitação exponencial}

A distribuição exponencial é comumente utilizada em conjuntos de dados com forte assimetria (forma de "J" invertido), sendo aplicada preferencialmente em série de dados de períodos secos (NETO et al., 2005). A sua função é descrita na equação 4 (KITE, 1978):

$$
\begin{aligned}
& f(x)=\lambda e^{-\lambda X} ;(X>0) \\
& f(x)=0 ;(X \leq 0)
\end{aligned}
$$$$
\text { equação } 4
$$

A função de distribuição acumulada é do tipo: 


$$
F(x)=P(X \geq 0)=\int_{0}^{\infty} f(x) d x=1-e^{-\ell x}=1-\alpha
$$

equação 5

Sendo que o parâmetro de distribuição $(\lambda)$ é estimado pelo inverso da média.

A função densidade de probabilidade é apresentada de diversas formas, sendo comumente apresentada na forma da equação 6 :

$$
\begin{gathered}
F(x)=\frac{\gamma}{\beta}\left(\frac{x-\alpha}{\beta}\right)^{\gamma-1} \exp \left[-\left(\frac{x-\alpha}{\beta}\right)\right]^{\gamma} ;(X e \geq \alpha) \quad \text { equação } 6 \\
f(x)=0 ;(X<\alpha)
\end{gathered}
$$

onde: $\mathrm{X}$ é a precipitação pluvial, $\alpha, \beta$ e $\gamma$ são parâmetros empíricos da distribuição. Esta equação é denominada de distribuição de Weibull com três parâmetros e a sua distribuição acumulada é dada por:

$$
F(x)=P(X \geq 0)=\int_{0}^{\infty} f(x) d x=1-\exp \left[-\left(\frac{x-\alpha}{\beta}\right)\right]^{\gamma}=1-\alpha \quad \text { equação } 7
$$

onde: a é chamada de parâmetro de posição, pois controla a posição da curva sobre o eixo da abscissa. $O$ parâmetro de escala $\beta$ controla as dimensões que a curva assume, dada uma forma constante e $\gamma$ é denominado de parâmetro de forma (NETO et al. 2005).

\subsection{Distribuição Weibull}

A distribuição Weibull é utilizada em análise hidrológica para eventos extremos. Essa distribuição foi proposta primeiramente por Fisher e Tippet em 1928, tendo sido desenvolvida independentemente por Walodi Webull, em 1939 (NETO et al. 2005). O principal método de ajuste da distribuição de Webull é através da máxima verossimilhança (COUTO, 1980), onde os valores de $\gamma$ e $\beta$ são determinados através das suas equações fundamentais. Através da equação 9 pode-se verificar que $\beta$ é função de $\gamma$ e que a solução para este tipo de sistema é obtida através de um processo iterativo, como por exemplo o método da bissecção.

$$
\frac{\sum_{i=1}^{n} x_{i}^{\widehat{\gamma}} \ln \left(x_{i}\right)}{\sum_{i=1}^{n} x_{i}}-\frac{\sum_{i=1}^{n} \ln \left(x_{i}\right)}{n}-\frac{1}{\widehat{\gamma}}=0
$$


Após a aplicação da distribuição Gama, Log-normal, Exponencial ou Weibull, normalmente utiliza-se o teste de aderência de Kolmogorov-Smirnov ou teste de $\chi$ quadrado, a $5 \%$ de significância, para verificar o ajuste entre os dados estimados e os observados (ASSIS, ARRUDA E PEREIRA, 1996). O teste de Kolmogorov-Smirnov é baseado no módulo da maior diferença entre a probabilidade observada e a estimada, que é comparada com um valor tabelado de acordo com o número de observações da série que está em teste (CATALUNHA et al., 2002). No teste de $\chi$-quadrado a hipótese de nulidade admite que a distribuição seja aquela especificada com os seus parâmetros estimados baseados em seus dados amostrais.

\section{REDES NEURAIS ARTIFICIAIS}

As Redes Neurais Artificiais (RNA) são conjuntos de modelos matemáticos capazes de simular o sistema neurológico humano. Esta técnica vem sendo utilizada nas mais diversas áreas do conhecimento, para a resolução de diversos tipos de problemas, como por exemplo: a aproximação de funções, a previsão de séries temporais e o reconhecimento de padrões. Uma das grandes vantagens das RNAs é que na maioria dos casos, as soluções possuem uma eficácia igual ou superior a das outras técnicas.

De uma forma simples, a RNA pode ser definida como uma ferramenta estatística, que, por meio do processamento de informações, gera uma saída (dados preditos) a partir de uma ou mais entradas (preditores). Através de sucessivas apresentações dos dados de entrada e saída previamente conhecidas, a RNA aprende a relação entre elas (entrada e saída) e através de um algoritmo de minimização de erros, procura reduzir o erro quadrático médio em cada iteração do treinamento.

Uma Rede Neural é formada por elementos processadores simples. Cada elemento processador executa uma função simples, mas a RNA como um todo tem capacidade computacional para resolução de problemas complexos. A estrutura apresentada na Figura 1 possui quatro entradas $\left(x_{1}, x_{2}, x_{3}, x_{4}\right)$, duas saídas $\left(y_{1}, y_{2}\right)$ e quatro neurônios na camada intermediária, e é capaz de resolver problemas de regressão, 
classificação ou predição no espaço $R^{4}$. É também um exemplo de rede neural do tipo alimentada adiante ou feedfoward (BRAGA, CARVALHO e LUDEMIR, 2000).

Figura 1: Esquema de uma rede com duas (2) camadas.

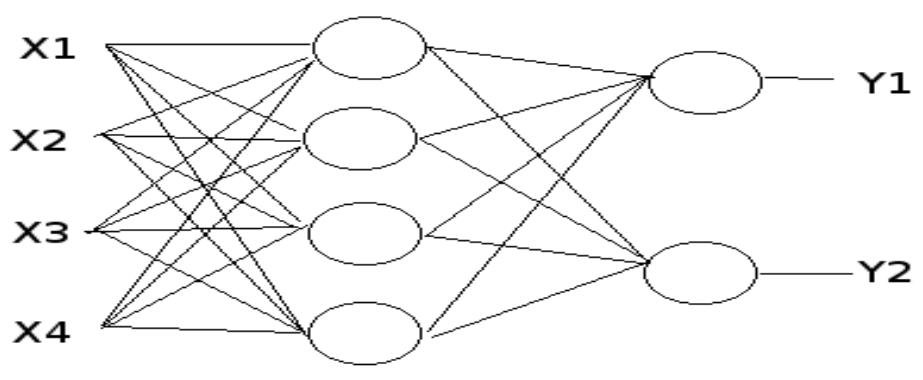

Fonte: KOVÁCS, Zsolt L. (1996).

As redes neurais artificiais caracterizam-se pelo aprendizado realizado através de exemplos. Para um determinado conjunto de dados, o algoritmo de aprendizado deve ser responsável pela adaptação dos parâmetros da rede, de maneira que, em um número finito de interações do algoritmo, haja convergência para uma solução. $O$ critério de convergência varia de acordo com o algoritmo e com o paradigma de aprendizado, mas pode envolver, por exemplo, a minimização de uma função objetivo, a variação do erro de saída ou mesmo a variação das magnitudes dos vetores de peso da rede (BRAGA, CARVALHO e LUDEMIR, 2000).

Basicamente, considera-se que o processo de aprendizado tem como característica a ocorrência de estímulo da rede pelo meio externo através da apresentação do conjunto de dados. Como consequência deste estímulo, o algoritmo de aprendizado provoca mudança nos parâmetros da rede e, finalmente, a mudança nos parâmetros acarreta em mudança no comportamento da RNA. Espera-se que a mudança gradual no comportamento da rede resulte em melhoria gradativa do seu desempenho. A modificação do processamento ou da estrutura de conhecimento de uma rede neural envolve a alteração do seu padrão de interconexão (KOVÁCS, 1996). Em princípio, isto pode ser feito de três maneiras: i) desenvolvimento de novas conexões; ii) perda de conexões existentes na rede ou; iii) modificação dos pesos das conexões já existentes.

Segundo BRAGA, CARVALHO e LUDEMIR (2000), "o objetivo principal do aprendizado em Redes Neurais é a obtenção de modelos com boa capacidade de 
generalização tendo como base o conjunto de dados”. Em problemas de aproximação, classificação e predição, o conjunto de treinamento é composto por pares de entrada e saída $\left(x, y_{d}\right)$, sendo caracterizada previamente a saída desejada $y_{d}$ para um determinado valor de entrada $x$. O ajuste de pesos deve modificar a saída $y$ de forma que a diferença entre $y$ e $y_{d}$, ou seja, o erro seja reduzido a cada interação. No entanto, a minimização do erro pode não levar a resultados satisfatórios. Desta forma, é necessário mais do que uma simples minimização do erro do conjunto de treinamento para obter uma boa resposta de generalização. O objetivo dos algoritmos de treinamento deve ser o de aproximar funções geradoras $f_{g}$ dos dados e não simplesmente o de minimizar o erro do conjunto de treinamento.

Para esse aprendizado, podem ser empregados os seguintes métodos:

- Aprendizado supervisionado: neste método a rede deve ser capaz de medir a diferença entre seu comportamento atual e o comportamento de referência e então corrigir os pesos de maneira a reduzir este erro.

- Aprendizado não supervisionado: os pesos da rede são modificados em função de critérios internos como, por exemplo, a repetição de padrões de ativação em paralelo de vários neurônios. O comportamento resultante deste tipo de aprendizado é usualmente comparado com técnicas de análise de dados empregadas na estatística.

- $\quad$ Aprendizado por competição: nesse caso é dado um padrão de entrada para fazer com que as unidades de saída disputem entre si para serem ativadas. Neste modelo existe uma competição entre as unidades de saída para decidir qual delas será a vencedora, e que consequentemente, terá a sua saída ativada e seus pesos atualizados no treinamento.

- Aprendizado por reforço: esse pode ser visto com um caso particular de aprendizado supervisionado. A principal diferença entre o supervisionado clássico e o aprendizado por reforço é a média de desempenho usada em cada um dos sistemas.

O Perceptron de Múltiplas Camadas é uma generalização da rede Perceptron proposto por Rosenblatt, onde cada neurônio da camada antecedente se conecta com todos os neurônios artificiais da camada seguinte e assim por diante. A rede é treinada de forma supervisionada, usando o algoritmo de retro-propagação do erro fundamentado na regra delta Adaline generalizada, que ajusta os pesos sinápticos através de estimativas do vetor do erro em relação aos pesos sinápticos. 
O MLP é uma rede cuja topologia constitui-se de uma camada sensorial ou de entrada, que possui tantos nós de entrada quantos forem os sinais de entrada, que dependem intrinsecamente do problema, uma ou mais camadas intermediárias ou ocultas de neurônios e uma camada de saída com um número de neurônios idênticos ao número de sinais de saída, que são tantos quantos forem necessários para a modelagem do problema. O sinal de entrada se propaga para frente (feedforward) através das camadas ocultas, até a camada de saída. Portanto, o MLP é uma rede alimentada para frente ou rede alimentada adiante (HAYKIN, 2001).

O número de camadas ocultas e a quantidade de neurônios artificiais, de cada uma dessas camadas, dependem da complexidade do problema que se deseja solucionar. A figura 2 mostra a arquitetura de um MLP, onde $X_{i}, X_{h}$ e $X_{n}$ correspondem respectivamente, ao número de elementos dos vetores da camada de entrada, da camada oculta e da camada de saída.

Os elementos $X_{i}, X_{h}$ e $X_{n}$ correspondem, respectivamente, aos vetores dos sinais de entrada da RNA, e das saídas desejadas impostas pelo "supervisor".

Figura 2: Modelo Multicamada Perceptron
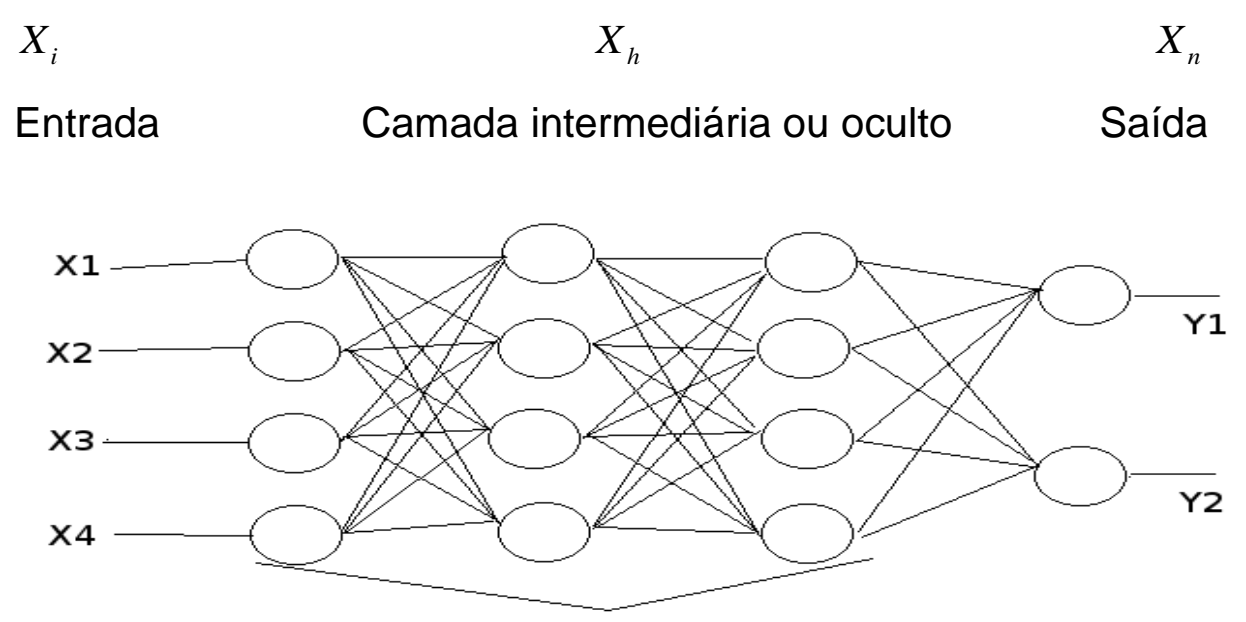

Fonte: BRAGA, P.B., CARVALHO, A.C.P.L.F. e LUDEMIR, T.B. (2000).

O treinamento da MLP, usando o algoritmo de retro-propagação do erro, é realizado em duas etapas: a primeira é chamada de propagação e a segunda de retropropagação (ARTERO, 2009). Durante a primeira etapa do treinamento o vetor de sinais de entrada $X_{i}=\left[X_{1}, X_{2}, X_{3}, X_{4}, \cdots, X_{n}\right]^{T}$ é aplicado a cada neurônio da primeira camada oculta da rede, cada qual calculando a sua respectiva saída, que é então transmitida para 
frente como sinal de entrada para todos os neurônios da camada oculta subsequente $X_{h}$, que repetem o mesmo procedimento de cálculo e transmissão, e assim por diante até a camada de saída da rede, quando o valor da saída obtida pela rede $X_{n}=\left[X_{1}, X_{2}, \ldots, X_{n}\right]^{T}$ é comparado com o valor da saída desejada $S$ (saída), obtendo-se o sinal de erro $e_{j}=T_{j}-S_{j}$ de cada neurônio $j$ desta camada para cada padrão apresentado.

O erro médio quadrático, considerando todos os $X_{n}$ neurônios da camada de saída $j$ e após a apresentação de todos os padrões de treinamento, completando assim um período é calculado e definido por,

$$
E=\frac{1}{Q} \sum_{q=1}^{Q} E^{q}=\frac{1}{Q} \sum_{q=1}^{Q} \cdot \frac{1}{2} \sum_{j=1}^{N_{O}}\left(e_{j}\right)^{2}=\frac{1}{Q} \sum_{q=1}^{Q} \cdot \frac{1}{2} \sum_{j=1}^{N_{O}}\left(T_{j}-O_{j}\right)^{2} \quad \text { equação } 10
$$

onde: $O_{j}$ e $T_{j}$ são respectivamente a saída calculada e a saída desejada para o neurônio $j$, onde j $=1,2, \ldots N_{0}$ e $E^{q}$ é o erro total instantâneo no padrão $q$ (KOVÁCS, 1996).

A segunda etapa ocorre em sentido contrário. Há uma retro-propagação do sinal de erro calculando-se $\delta$ de cada neurônio, atualizando os pesos das conexões a partir da camada de saída $j$, propagando-se recursivamente pelas camadas ocultas até a camada de entrada, pelo uso da regra delta generalizada representada pela equação 11.

$$
\Delta W_{j i}=-N \cdot \frac{(\partial E)}{\left(\partial W_{j i}\right)}
$$

onde: $\boldsymbol{N}$ é a taxa de aprendizado e $\boldsymbol{i}$ indica indexação referente à camada anterior $\boldsymbol{I}$.

Verifica-se na equação acima que o algoritmo BP, calcula as derivadas parciais de uma função de erro $\boldsymbol{E}$, com relação ao vetor peso $\boldsymbol{W}$, de certo vetor de entrada $\boldsymbol{X}$. O algoritmo de BP, apesar de eficiente em várias aplicações pode apresentar demora ao longo tempo da convergência.

A taxa de aprendizado $\boldsymbol{N}$ é que responde à velocidade com que ocorre a busca no espaço dos pesos, em direção aos valores que resultam em um erro global mínimo. Se a taxa de aprendizado não for adequadamente escolhida, podem ocorrer problemas durante o treinamento da rede. Quanto menor o valor de $\mathbf{N}$ mais suave será a curva de treinamento, porém o aprendizado se tornará muito lento podendo, inclusive, ocorrer uma 
paralisia da rede em pontos onde a derivada da função de ativação seja nula ou infinitamente pequena.

O treinamento onde a taxa de aprendizado é muito baixa, com uma variação muito pequena de $\Delta W_{j i}$ um cálculo para outro, provoca uma suave trajetória no espaço dos pesos, aumentando as chances de a rede ficar presa em um mínimo local, ou, na melhor das hipóteses, o treinamento ficar muito lento. Em contrapartida, se for usado um valor muito alto para a taxa de aprendizado, ocorrerão modificações muito intensas nos pesos, devido a uma alta variação de $\Delta W_{j i}$ provocando oscilações intensas na curva de treinamento, causando instabilidade na rede.

O problema da ocorrência de oscilações na curva de treinamento da RNA devido à utilização de altas taxas de aprendizado pode ser bastante amenizado através do uso de Coeficiente de Momento (momentum) na regra delta, de acordo com (BRAGA, 2000), assim o termo momentum passa a ser representada pela equação 12.

$$
W_{j i}(T+1)=W_{j i}+N \sigma_{j}(t) x_{i}(t)+\alpha\left(W_{i j}(t)-W_{i j}(T-1)\right) \quad \text { equação } 12
$$

Tal equação tem a função básica de identificar qual o melhor momento de parar ou interromper o ciclo de aprendizado da rede neural artificial, contribuindo assim para a generalização da rede.

O uso de RNAs como modelos de previsão meteorológicas foram usadas em vários estudos, dentre os quais se pode citar ASCE, 2000; RAMIREZ, VELHO e FERREIRA, 2005; SRIVASTAVA et al., 2010; TALEI, CHUA e QUEK, 2010 e WU, CHAU e FAN, 2010, mostrando seu potencial nesta área de estudo.

Zhang et al. (1997) construíram um sistema especialista baseado na teoria dos grupos de redes neurais para a predição de chuva que foi testado no National Oceanic and Atmospheric Administration (US Department of Commerce). Este modelo conseguiu reduzir a margem de erro de $30 \%$ para $10 \%$.

\section{CONSIDERAÇÕES FINAIS}

Tanto os modelos matemáticos probabilísticos, bem como as redes neurais artificiais são modelos alternativos à tarefa de predição, geralmente aplicadas na 
modelagem de séries complexas. Dependendo da região de onde os dados foram coletados, um modelo matemático probabilístico se adequa melhor em relação ao outro. Geralmente, testam-se alguns modelos e o que melhor prediz a precipitação na referida região é utilizado. Ao se utilizar as redes neurais artificiais, para que se obtenham resultados válidos é preciso compreender as diferentes formas de construir e aplicar a RNA. Deve-se analisar as especificidades de cada rede e avaliar qual melhor se adequa à modelagem temporal. Ao se tomar as devidas precauções podem-se criar modelos capazes de realizar a predição de precipitação com um bom nível de acerto, tanto através dos modelos matemáticos probabilísticos bem como através das RNAs, o que pode auxiliar a população local.

\section{REFERÊNCIAS}

ARTERO, A. O. Inteligência Artificial: Teoria e Prática, São Paulo: Editora Livraria da Física, 2009.

ASCE Task Committee on application of artificial neural networks in hydrology. Artificial neural networks in hydrology. II: Hydrologic application. Journal of Hydrologic Engineering, Reston, v.5, p.124-137, abr. 2000.

ASSIS, F. N.; ARRUDA, H. V.; PEREIRA, A. R. Aplicações de estatística à climatologia. Pelotas: Universitária, 1996.

ÁVILA, L.F.; de MELLO, C.R.; VIOLA, M.R. Mapeamento da precipitação mínima provável para o sul de Minas Gerais. Revista Brasileira de Engenharia Agrícola e Ambiental, Campina Grande, v.13, (Suplemento), p.906-915, dez. 2009.

BRAGA, A. P., CARVALHO, A.C.P.L.F.; LUDEMIR, T.B. Redes neurais artificiais: teoria e aplicações, Rio de Janeiro: Livros Técnicos e Científicos, 2000.

CATALUNHA, M.J.; SEDIYAMA, G.C.; LEAL, B.G.; SOARES, C.P.; RIBEIRO, A.B. Aplicação de cinco funções densidade de probabilidade a séries de precipitação pluvial no 
Estado de Minas Gerais. Revista Brasileira de Agrometeorologia, Santa Maria, v.10, n.1, p.153-162, jan. 2002.

Couto, H.T.Z. Distribuição de diâmetro em plantações de Pinus caribea Morelet, 1980, 79p. Tese (Livre Docência), Escola Superior de Agricultura “Luiz de Queiroz"/USP, Piracicaba.

CRUCIANI, D.E; MACHADO, R.E.; SENTELHAS, P.C. Modelos da distribuição temporal de chuvas intensas em Piracicaba, SP. Revista Brasileira de Engenharia Agrícola e Ambiental, Campina Grande, v.6, n.1, p.76-82, jan./abr. 2002.

CUNHA. A.R.; MARTINS, D.; PASSOS, J.R.S. O modelo de probabilidade aplicado ao estudo da distribuição da chuva na região administrativa de Bauru, SP. In: Congresso Brasileiro de Engenharia Agrícola, 1996, Bauru. Anais... Bauru SBEA, 1996, p.1-7.

HAYKIN, S. S. Redes Neurais: Princípios e Práticas. 2.ed., Porto Alegre: Bookman, 2001.

HOLMAN, K.D.; VAVRUS, S.J. Understanding simulated extreme precipitation events in Madison, Wisconsin, and therole of moisture flux convergence during the late twentiethand twenty-first centuries. Journal of Hydrometeorology, Washington, v.13, n.3, p.877-894, jun. 2012.

KATTSOV, V.M.; WALSH, J.E.; CHAPMAN, W.L.; GOVORKOVA, V.A.; PAVLOVA, T.V.; ZHANG, X. Simulation and projection of Arctic freshwater budget components by the IPCC AR4 global climate models. Journal of Hydrometeorology, Washington, v.8, n.3, p.571589, jun. 2007.

KAWAZOE, S.; GUTOWSKIM Jr., W.J. Regional, Very Heavy Daily Precipitation in NARCCAP Simulations, Journal of Hydrometeorology, Washington, v.14, n.4, p.12121227, ago. 2013.

Kite, G.W. Frequency and risk analysis in hydrology, Fort Collins: Water Resources Publications, 1977. 
KOVÁCS, Z. H. Redes neurais artificiais: fundamentos e aplicações, 2 ed., São Paulo: Editora Acadêmica, 1996.

LIMA, J.S.S.; SILVA, S.A.; OLIVEIRA, R.B.; CECÍLIO, R.A.; XAVIER, A.C. Variabilidade temporal daprecipitação mensal em Alegre - ES. Revista Ciência Agronômica, Fortaleza, v.39, n.2, p.327-332, abr./jun. 2008.

MOREIRA, P.S.P.; DALLACORT, R.; MAGALHÃES, R.A.; INOUE, M.H.; STIELER, M.C.; DA SILVA, D.J.; MARTINS, J.A. Distribuição e probabilidade de ocorrência de chuvas no município de Nova Maringá - MT. Revista de Ciências Agro-Ambientais, Alta Floresta, v.8, n.1, p.9-20, 2010.

MURTA, R.M.; TEODORO, S.M.; BONOMO, P.; CHAVES, M.A. Precipitação pluvial mensal em níveisde probabilidade pela distribuição gama para duas localidades do sudoeste da Bahia. Ciência e Agrotecnologia, Lavras, v.29, n.5, p.988-994, set./out. 2005.

NETO, D.D.; de ASSIS, J.P.; TIMM, L.C.; MANFRON, P.A.; SPAROVEK, G.; MARTIN, T.N. Ajuste de modelos de distribuição de probabilidade a séries históricas de precipitação pluvial diária em Piracicaba-SP. Revista Brasileira de Agrometeorologia, v.13, n.2, p.273-283, mai./ago. 2005.

QUEIROZ, E.F.; SILVA, R.J.B.; OLIVEIRA, M.C.N. Modelo de análise de regressão periódica daprecipitação mensal, da bacia atlântico sudeste, no Estado do Paraná, Pesquisa Agropecuária Brasileira, Brasília, v.36, n.5, p.727-742, mai. 2001.

RODRIGUES, L.N.; PRUSKI, F.F. Precipitação provável para João Pinheiro, Minas Gerais, utilizando as funções de distribuição de probabilidades gama e log-normal. Revista Brasileira de Engenharia Agrícola e Ambiental, Campina Grande, vol.5, n.3, p. 563-567, set./dez. 2001. 
SAMPAIO, S.C.; CORRÊA, M.M. SOUZA, M.R.; GUIMARÃES, J.C.; SILVA, A.M. Precipitação provável para o município de Lavras, MG, utilizando a distribuição lognormal. Revista Ciência e Agrotecnologia, Lavras, v.23, n.2, p.382-398, abr./jun. 1999.

SAMPAIO, S.C.; de QUEIROZ, M.M.F.; FRIGO, E.P.; LONGO, A.J.; SUSZEK, M. Estimativa e distribuição de precipitação decendiais para o estado do Paraná, Irriga, Botucatu, v.12, n.1, p.38-53, jan./mar. 2007.

SILVA, J.C.; HELDWEIN, A.B.; MARTINS, F.B.; TRENTIN, G.; GRIMM, E.L. Análise de distribuiçãode chuva para Santa Maria, RS. Revista Brasileira de Engenharia Agrícola e Ambiental, Campina Grande, v.11, n.1, p.67-72, fev. 2007.

SRIVASTAVA, G.; PANDA, S.N.; MONDAL, P.; LIU, J. Forecasting of rainfall using oceanatmospheric indices with a fuzzy neural technique. Journal of Hydrology, v.392, n.3-4, p. 190-198, out. 2010.

TALEI, A.; CHUA, L. H. C.; QUEK, C.A. Novel Application of a Neuro-Fuzzy Computational Technique in Event-Based Rainfall-Runoff Modeling. Expert Systems with Applications, Amsterdam, v. 37, n.12, p. 7456-7468, dez. 2010.

THOM, H.C.S. A note on the gama distribution. Monthly Weather Review, Washington, v.86, n.4, p.117-122, abr. 1958.

RAMIREZ, M.C.V., VELHO, H.C.; FERREIRA, N.J. Artificial neural network technique for rainfall forecasting applied to the São Paulo region. Journal of Hydrology, v.301, n.1-4, p. 146-162, jan. 2005.

WU, C. L.; CHAU, K. W.; FAN, C. Prediction of Rainfall Time Series Using Modular Artificial Neural Networks Coupled with Data-Preprocessing Techniques. Journal of Hydrology, v.389, N.1-2, p. 146-167, jul. 2010. 
ZHANG, M.; FULCHER, J.; SCOFIELD, R. A. Rainfall estimation using artificial neural network group. Neurocomputing, New York, v.16, n.2, p.97-115, jul. 1997. 